\title{
Analysis on the Benefits and Risk Aversion Strategies of the Construction of Production, Teaching and Training Bases in Higher Vocational Colleges- Take the Construction of Jiangsu Traditional Chinese Medicine Industry Science and Education Park as an Example
}

\author{
DUAN Qiong-hui ${ }^{1}$, LI Yong ${ }^{*}, 2$ \\ ${ }^{1,2}$ Jiangsu Agri-animal Husbandry Vocational College, China
}

*Corresponding Author: LI Yong, Jiangsu Agri-animal Husbandry Vocational College, China

\begin{abstract}
Occupation and practicality are the basic characteristics of higher vocational education. therefore, higher vocational colleges pay special attention to the construction of practical training hardware in schools. Although the original in-school practice training rooms and laboratories of the higher vocational colleges are still the main places for students to practice and practice, with the rapid development of higher vocational education, especially the development requirements of the integration of production and teaching, the construction of the integration base of production and teaching inside and outside the colleges is in full swing in the development of the higher vocational colleges. This paper uses the method of literature analysis, combining with the construction practice of Jiangsu traditional Chinese medicine teaching park, which is the production and teaching training base of Jiangsu vocational college of agriculture and animal husbandry science and technology, to analyze the comprehensive benefits, risks and countermeasures of the construction of the production and teaching integration base in higher vocational colleges, hoping to provide useful reference and reference for the construction of the production and teaching integration base in the future.
\end{abstract}

Keywords: Higher vocational colleges; Teaching and training base; Benefits; Risks; Strategy

\section{INTRODUCTION}

The state Council's decision on the development of modern vocational education pointed out: " by 2020, a modern vocational education system with Chinese characteristics and world level will be formed that meets the needs of development, integrates production and education in depth, connects vocational colleges, communicates vocational education with general education, and embodies the concept of lifelong education. " the integration of industry and education and school-enterprise cooperation are the main demands of higher vocational education for a long period of time in the future. they are important starting points for vocational colleges to improve the quality of education and teaching and personnel training. they are also programmatic documents for the construction of a modern vocational education system and for the absorption of in-depth participation of industries, trades and enterprises in vocational education. At the same time, in recent years, with the enhancement of residents' health awareness and the increasing influence of traditional Chinese medicine in various countries all over the world, the traditional Chinese medicine industry is also booming, and the demand for training talents of traditional Chinese medicine is also increasing. various related higher vocational colleges have set up related specialties of traditional Chinese medicine to vigorously train outstanding graduates of traditional Chinese medicine. Jiangsu institute of agriculture and animal husbandry, focusing on the needs of Taizhou's regional economic development, has set up the specialty of traditional Chinese medicine in due course, vigorously trained graduates of the specialty of traditional Chinese medicine, and trained many high-quality technical and skilled talents for the Chinese medicine city.

The deep integration of production and education in vocational education is an important way to construct the modern vocational education system and realize the coordinated development of vocational education and regional economy and society. The construction of Jiangsu traditional 
Analysis on the Benefits and Risk Aversion Strategies of the Construction of Production, Teaching and Training Bases in Higher Vocational Colleges- Take the Construction of Jiangsu Traditional Chinese Medicine Industry Science and Education Park as an Example

Chinese medicine science and technology teaching park, an experimental training base for in-depth integration of production and teaching, is an inevitable choice for Jiangsu agricultural science and technology vocational college to adapt to the development of the situation, improve the teaching quality and personnel training level of vocational education, and serve the local economy. it is an urgent requirement for the college's own development and an effective way to promote the extension and broadening of the depth and breadth of school-enterprise cooperation in vocational education ${ }^{[1]}$.

The government, schools, enterprises, industries and other parties have started to invest in the construction of training bases and build a deep integration base of production and teaching. the implementation of production and teaching depth and school-enterprise cooperation is an important measure, which is of great significance for carrying out modern apprenticeship teaching and improving the quality of personnel training. However, the construction of the training base presents the trend of diversification of subjects, diversification of functions and multi-level benefits ${ }^{[2]}$. although it has good social and ecological benefits, it also has higher risks. Before the construction, it is necessary to establish reasonable risk aversion measures to minimize risks, attract the investment of capital, manpower and material resources of industries and enterprises, and allow enterprises and industries to participate in the entire process of base construction and personnel training. Taking the construction of Jiangsu traditional Chinese medicine industry science and education park as an example, this paper analyzes the main benefits of the training base, evaluates the main risks existing, and puts forward some countermeasures for reference.

\section{Analysis Of Main Benefits}

\subsection{Social Benefits}

Jiangsu traditional Chinese medicine industry science and education park is an organic combination of scientific research, production, practical training, demonstration, promotion and ecological sightseeing. it is deeply integrated with China's medical city, expanding and improving its functions, optimizing the agricultural structure of Gao-gang district, enriching and upgrading the scientific research and technical promotion and personnel training base of Jiangsu vocational college of agriculture and animal husbandry science and technology, and adding new features and leisure places for tourists. At the same time, with the gradual improvement of the construction and scale of the traditional Chinese medicine science and education park, it has begun to produce certain social influence. bi will add a brilliant stroke to the rapid development of Taizhou pharmaceutical industry. Therefore, the production-teaching integration base has very significant social benefits.

\subsection{Economic Benefits}

The traditional Chinese medicine industry is a traditional industry in our country. in recent years, the demand for Chinese medicinal materials has been increasing year by year both at home and abroad. the price continues to rise. the demand for Chinese medicinal materials by several large pharmaceutical enterprises represented by Yang-zijiang pharmaceutical industry in Taizhou continues to increase. The construction of this base is the deep integration of education and industry, which can drive the cultivation of villages and towns around the construction site, especially the cultivation of high value-added Chinese herbal medicines, and will bring considerable economic benefits to the vast number of planting farmers in the surrounding rural areas. At the same time, the construction unit will also benefit from the demonstration and promotion.

\subsection{Ecological Benefits}

The construction of the base will not bring environmental pollution to the surrounding environment. upon completion, the ecological landscape of " garden full of flowers and fragrance in all seasons" will become a dazzling bright spot in Taizhou region with obvious ecological benefits. Jiangsu traditional Chinese medicine science and education garden is based on traditional Chinese herbal medicine resources and integrates with modern garden design elements. with the help of traditional Chinese herbal medicines such as coptis root of bitter medicine, lotus seed of clear water lotus, folium artemisiaeargyi of dragon boat festival, and cornel chrysanthemum of Chongyang, it conveys rich traditional Chinese medicine cultural characteristics and at the same time abides by the principles of " scientific planning, classified guidance, local conditions, economic application, and sustainable 
Analysis on the Benefits and Risk Aversion Strategies of the Construction of Production, Teaching and Training Bases in Higher Vocational Colleges- Take the Construction of Jiangsu Traditional Chinese Medicine Industry Science and Education Park as an Example

development" of modern ecological garden design. it creates garden landscape effects such as " trees and shrubs alternate with each other, height and height matching, evergreen and deciduous complementation" ${ }^{[4-5]}$, presenting a " domestic first-class" ecological garden of traditional Chinese medicine culture, which not only demonstrates the rich traditional Chinese medicine of " tiger keeps apricot forest."

\subsection{Teaching Benefits}

The implementation of the training project of the production, teaching and integration base can provide a wide range of training places for students of related specialties such as Chinese medicine pharmacy, landscape design, gardening, etc. it can also provide a scientific research platform for teachers, especially full-time teachers such as animal medicine college and landscape science and technology college, and provide professional quality education and training places for students of other secondary colleges of the college, as well as landscape design places for students of landscape science and technology related majors. Therefore, upon completion, it will be a beneficial carrier for the implementation of modern apprenticeship system, the implementation of work-study combination and the in-depth integration of production and teaching, and will have good teaching benefits ${ }^{[6-7]}$.

\section{Risk ASSESSMENT AND AVOIDANCE MEASURES}

The construction of the training base project of the Chinese medicine industry science and education park is a high-input and high-output industry. Although the country has been strongly supporting the development of the traditional Chinese medicine industry, due to various reasons, the project still has certain risks, mainly in the following aspects.

\subsection{Market Risk}

The important basis for the construction of this project is market information, including supply and demand information, price information, world medical development trend, etc. of the Chinese herbal medicine market. The basis of the project construction plan is the current market information and forecast of the future market. In today's economic globalization and marketization, if the future market development is not consistent with the predicted results in the project, it will bring greater risks.

Measures to avoid market risks: the park will set up a special market information investigation team to timely and accurately grasp local, national and even world market information on traditional Chinese medicine planting to prevent short-term market supply and demand imbalance and price decline caused by following the trend of planting. The market information research team should provide timely scientific decision-making suggestions for the planting, processing and sale of Chinese herbal medicines, and strive to minimize the risks brought about by market changes.

\subsection{Technical Risks}

Both traditional Chinese medicine planting and ecological cultivation in the park have high scientific and technological content. If the technical service fails to keep up with it, it will reduce its production performance, limit its normal economic benefits and create risks in the production process.

Measures to avoid technical risks: adhere to the people-oriented principle, fully mobilize the enthusiasm of employees, and introduce experts with genuine knowledge. To strengthen the joint and cooperation with peer experts and institutions of higher learning, to jointly carry out personnel training and scientific research, to develop new varieties, new products and new technologies, to strengthen technology promotion, and to effectively avoid risks.

\subsection{Decision-Making Risks}

The risk of decision-making refers to the loss caused by production operators' mistakes in decisionmaking during the implementation of the project. In the process of planning and implementation, it may be because the information is inaccurate or misleading, or it may also be because the production operators lack the scientific decision-making ability and lead to decision-making mistakes. There is also a possibility that the competent leader will make mistakes due to his hasty and subjective decision-making in advancing the project process through administrative means. 
Analysis on the Benefits and Risk Aversion Strategies of the Construction of Production, Teaching and Training Bases in Higher Vocational Colleges- Take the Construction of Jiangsu Traditional Chinese Medicine Industry Science and Education Park as an Example

Measures to avoid decision-making risks: establish effective channels so that price information, supply and demand information, and technical information can be in place in time. Decision makers continue to learn and improve the level of scientific decision - making.

\subsection{Credit Risk}

Credit risk refers to the loss caused to the park by the failure of the product purchase and sale contract between the base, enterprises and farmers to perform during the development of the park.

Measures to avoid credit risk: during the implementation of the project, to solve the sales problem of products, at the beginning of the production of traditional Chinese medicine, legal guarantee purchase and sale contracts were signed with farmers and traditional Chinese medicine production enterprises, and production was organized by " order form", " cooperative" and " entrusted cultivation".

\subsection{Production Risk}

The production risk is closely related to the project organization and management mode. good management mechanism and management mode are the most effective ways to avoid the production risk. In the process of agricultural industrialization in our country, practice has proved that the management mode of " company / enterprise + base + peasant household" is the key to solve the production risk.

In addition, natural disasters in agricultural production will also bring certain risks to the park: on the one hand, disastrous weather such as supercooling, overheating, drought and torrential rain will affect the performance of Chinese herbal medicines; On the other hand, insect pests, epidemic diseases and weeds will bring serious harm to the development of planting, breeding and processing industries, thus affecting normal production.

Effective measures to avoid natural risks are to do a good job of prevention and contact with meteorological departments in time to minimize losses caused by natural factors. Strengthen the prevention and control of plant diseases and insect pests and animal epidemic prevention. Strive for government support, perfect disaster relief system and introduce agricultural insurance services.

\section{CONCLUSiON}

The integration of production and education in higher vocational colleges undertakes the glorious mission of cultivating high-quality and high-skilled and application-oriented innovative talents. in fact, the training base is an important carrier and main platform for teachers in higher vocational colleges to carry out scientific research and production, management, serve the society, and cultivate high-end skilled talents on the frontline. it is also the main carrier to cooperate with theoretical teaching, carry out skill training and practical exercise, and embody occupational and technical skills. Therefore, it is of great strategic significance to construct and apply a good training base for the integration of production, teaching and training, which is highly valued by all higher vocational colleges.

\section{ACKNOWLEDGMENTS}

This research was financially supported by Jiangsu University Students Innovation and Entrepreneurship Training Program -School Enterprise Cooperation Fund Project (Grant NO: 201712806029H); The fifth phase of the "311 high level talent training project" in Taizhou (Grant NO: 2017-III-807); Jiangsu Agri-animal Husbandry Vocational College, Research on education and teaching research (Grant NO: JYZD201603; Grant NO: JYYB201603);"Phoenix talent project" of Jiangsu Agri-animal Husbandry Vocational College (Grant NO: 10434014001); and"the fourth session of the Chinese society of Vocational and technical education " financially support (Grant NO: 201610Y29).

\section{REFERENCES}

[1] XU xinwang. 2016, Path selection of deep integration of Vocational Education under the background of industrial transformation and upgrading. Journal of Higher Education, (24):13-15.

[2] Wang guihua. 2013, Research and Practice on long term mechanism of productive training base. Vocational \& Technical Education Forum (China). (12):82-85. 
Analysis on the Benefits and Risk Aversion Strategies of the Construction of Production, Teaching and Training Bases in Higher Vocational Colleges- Take the Construction of Jiangsu Traditional Chinese Medicine Industry Science and Education Park as an Example

[3] CHEN Sheng-bin, DU Shun-bao. 2008, The Plant Restoration and Sustainable Landscape Design for Jinshan Quarry Pits in Mudu Scenic Area. Chinese Landscape Architecture.4:74-77.

[4] Wang yanchen. 2018, Analysis of the functions and artistic techniques of plant landscaping in landscaping design. Xiandai Horticulture (China), (04): 95.

[5] Wei Jiaxing. 2017, Study on plant landscape in the campus of seven universities in Xianlin University in Nanjing.Hunan Agricultural Sciences, (01): 91-94.

[6] Chen Xiaojiang. An Analysis of How to Offer the Vocational Guidance by Internet in China[C]// International Conference on Management, Education and Social Science. 2017.

[7] Chen Xiaojiang. Research and Practice on Innovation and Entrepreneurship Education System in Vocational Colleges[C]// International Conference on Management, Education and Social Science. 2017.

Citation: DUAN Qiong-hui, LI Yong. "Analysis on the Benefits and Risk Aversion Strategies of the Construction of Production, Teaching and Training Bases in Higher Vocational Colleges- Take the Construction of Jiangsu Traditional Chinese Medicine Industry Science and Education Park as an Example". International Journal of Humanities Social Sciences and Education (IJHSSE), vol 5, no.9, 2018, pp. 16-20. doi: http://dx.doi.org/10.20431/2349-0381.0509003.

Copyright: () 2018 Authors. This is an open-access article distributed under the terms of the Creative Commons Attribution License, which permits unrestricted use, distribution, and reproduction in any medium, provided the original author and source are credited. 